\title{
Gene expression profiling predicts clinical outcome of prostate cancer
}

\author{
Gennadi V. Glinsky, ${ }^{1}$ Anna B. Glinskii, ${ }^{1}$ Andrew J. Stephenson, ${ }^{2}$ Robert M. Hoffman, ${ }^{3}$ \\ and William L. Gerald²
}

\author{
${ }^{1}$ Sidney Kimmel Cancer Center, San Diego, California, USA. ${ }^{2}$ Department of Pathology, Memorial Sloan-Kettering Cancer Center, New York, New York, USA. \\ ${ }^{3}$ AntiCancer Inc., San Diego, California, USA.
}

\begin{abstract}
One of the major problems in management of prostate cancer is the lack of reliable genetic markers predicting the clinical course of the disease. We analyzed expression profiles of 12,625 transcripts in prostate tumors from patients with distinct clinical outcomes after therapy as well as metastatic human prostate cancer xenografts in nude mice. We identified small clusters of genes discriminating recurrent versus nonrecurrent disease with $90 \%$ and $75 \%$ accuracy in two independent cohorts of patients. We examined one group of samples (21 tumors) to discover the recurrence predictor genes and then validated the predictive power of these genes in a different set ( 79 tumors). Kaplan-Meier analysis demonstrated that recurrence predictor signatures are highly informative $(P<0.0001)$ in stratification of patients into subgroups with distinct relapse-free survival after therapy. A gene expression-based recurrence predictor algorithm was informative in predicting the outcome in patients with early-stage disease, with either high or low preoperative prostate-specific antigen levels and provided additional value to the outcome prediction based on Gleason sum or multiparameter nomogram. Overall, $88 \%$ of patients with recurrence of prostate cancer within 1 year after therapy were correctly classified into the poor-prognosis group. The identified algorithm provides additional predictive value over conventional markers of outcome and appears suitable for stratification of prostate cancer patients at the time of diagnosis into subgroups with distinct survival probability after therapy.
\end{abstract}

\section{Introduction}

Critical clinical need in the development of reliable prognostic markers suitable for stratification of prostate cancer patients is clearly demonstrated by the results of a recent randomized study of the therapeutic efficacy of surgery versus watch-and-wait strategy, demonstrating only a modest $6.6 \%$ absolute reduction in mortality after prostatectomy compared with observation, despite the association of surgery with a $50 \%$ reduction in the hazard ratio for death from prostate cancer (1). It appears that a measurable clinical benefit of surgery is limited to a poorly defined subpopulation of prostate cancer patients; therefore, an improved ability to identify a subgroup of prostate cancer patients who would benefit from therapy should have a significant immediate positive clinical and socioeconomic impact. Widely used biochemical, histopathological, and clinical criteria such as prostate-specific antigen (PSA) level, Gleason score, the clinical tumor stage, and molecular genetic approaches assaying loss of tumor suppressors or gain of oncogenes (2) had only limited success with respect to prostate cancer patients' stratification and demonstrated a significant variability in predictive value among different clinical laboratories and hospitals. Furthermore, best existing markers cannot reliably identify at the time of diagnosis a poorprognosis group of prostate cancer patients who ultimately would fail therapy (3). Classification nomograms that incorporate measurements of several individual preoperative and postoperative parameters are generally recognized as the most efficient clinically useful models currently available for prediction of the probability of relapse-free survival after therapy of individual prostate cancer

Nonstandard abbreviations used: disease-free interval (DFI); phenotype association index (PAI); prostate-specific antigen (PSA); radical prostatectomy (RP).

Conflict of interest: Gennadi V. Glinsky is a named inventor on related patent applications.

Citation for this article: J. Clin. Invest. 113:913-923 (2004). doi:10.1172/JCI200420032. patients (4-7). One of the significant deficiencies of these classification systems, however, is that they have only limited utility in predicting the differences in outcomes readily observed between patients diagnosed with prostate cancers exhibiting similar clinical, histopathological, and biochemical features. Therefore, a critical clinical need exists to improve the classification accuracy of prostate cancer patients with respect to clinical outcome after therapy.

Expression profiling of prostate tumor samples using oligonucleotide or cDNA microarray technology revealed gene expression signatures associated with human prostate cancer (8-19), including potential prostate cancer prognosis markers $(9,14,16,17)$. One of the major limitations of these studies, however, was that the same clinical data set was used for both signature discovery and validation. Furthermore, usually only a single or few hits were validated using independent methods and independent clinical data sets, thus diminishing the potential advantage of the use of a panel of markers over a single marker in diagnostic and/or prognostic applications.

Here we applied a microarray-based gene expression-profiling approach to identify molecular signatures distinguishing subgroups of patients with differing outcomes and developed a stratification algorithm demonstrating high discrimination accuracy between subgroups of prostate cancer patients with distinct clinical outcomes after therapy using a training set of 21 prostate cancer patients. To validate a potential clinical utility of discovered genetic signatures, we confirmed the discrimination power of the proposed prostate cancer prognosis stratification algorithm using an independent set of 79 clinical tumor samples.

Our data suggest that identified molecular signatures have a significant potential for development of clinical prognostic tests suitable for stratification of prostate cancer patients at the time of diagnosis with respect to likelihood of negative or positive clinical outcome after therapy. Our results provide, to our knowledge, the first experimental evidence of a transcriptional resemblance between 
metastatic human prostate carcinoma xenografts in nude mice and primary prostate tumors from patients subsequently developing relapse after therapy. These data suggest that genetically defined metastasis-promoting features of primary tumors are a major contributing factor of aggressive clinical behavior and unfavorable prognosis in prostate cancer patients.

\section{Methods}

Clinical samples. In our experiments we used two independent sets of clinical samples for signature discovery (a training outcome set of 21 samples) and validation (a validation outcome set of 79 samples). Original gene expression profiles of the training set of 21 clinical samples (8 patients with recurrent prostate cancer defined as two successive PSA values greater than $0.2 \mathrm{ng} / \mathrm{ml}$ and 13 patients having remained disease-free for at least 4 years) analyzed in this study were recently reported (14). Primary gene expression data files of clinical samples as well as associated clinical information were provided by W. Sellers (Dana Farber Cancer Institute, Boston, Massachusetts, USA) and can be found at http://www-genome.wi.mit.edu/cancer/. The clinical and pathological features of the prostate cancer patients and prostate tumors analyzed in the previous study (14) were undistinguishable from those of 393 patients treated with radical prostatectomy (RP) during the sample collection period.

Prostate tumor tissues that comprise the validation data set were obtained from 79 prostate cancer patients undergoing therapeutic or diagnostic procedures performed as part of routine clinical management at Memorial Sloan-Kettering Cancer Center. Clinical and pathological features of 79 prostate cancer cases (37 patients with recurrent and 42 patients with nonrecurrent disease) constituting the validation outcome set are presented in Supplemental Table 1S (supplemental material available at http://www.jci.org/cgi/content/full/113/6/913/DC1). Median time of follow-up after therapy in this cohort of patients was 70 months. Samples were snap-frozen in liquid nitrogen and stored at $-80^{\circ} \mathrm{C}$. Each sample was examined histologically using H\&E-stained cryostat sections. Care was taken to remove non-neoplastic tissues from tumor samples. Cells of interest were manually dissected from the frozen block, and other tissues were trimmed away. All of the studies were conducted under Memorial Sloan-Kettering Cancer Center Institutional Review Board-approved protocols.

Cell culture. Cell lines used in this study were previously described (19) and are listed in Supplemental Table 2S. The LNCap-derived and PC-3-derived cell lines were developed by consecutive serial orthotopic implantation, either from metastases to the lymph node, or reimplanted from the prostate. This procedure generated cell variants with differing tumorigenicity, frequency, and latency of regional lymph node metastasis (19). Except where noted, cell lines were grown in RPMI-1640 supplemented with 10\% FBS and gentamycin (GIBCO BRL; Life Technologies Inc., Gaithersburg, Maryland, USA) to 70-80\% confluence and subjected to serum starvation as described (19) or maintained in fresh complete media supplemented with 10\% FBS.

Orthotopic xenografts. Orthotopic xenografts of human prostate PC-3 cells and sublines used in this study were developed by surgical orthotopic implantation as previously described (19). Briefly, $2 \times 10^{6} \mathrm{cul}-$ tured PC-3 cells, PC-3M or PC3-MLN4 sublines were injected subcutaneously into male athymic mice and allowed to develop into firm, palpable, and visible tumors over the course of 2-4 weeks. Intact tissue was harvested from a single subcutaneous tumor and surgically implanted in the ventral lateral lobes of the prostate gland in a series of six athymic mice per cell line subtype. The mice were examined periodically for suprapubic masses, which appeared faster in the PC3MLN4 than in the PC-3M sublines and somewhat faster in the PC-3M than in the PC-3 sublines. Tumor-bearing mice were sacrificed by $\mathrm{CO}_{2}$ inhalation over dry ice, and necropsy was carried out in a $2-4^{\circ} \mathrm{C}$ cold room. Typically, bilaterally symmetric prostate gland tumors in the shape of greatly distended prostate glands were apparent. Prostate tumor tissue was excised and snap-frozen in liquid nitrogen. The elapsed time from sacrifice to snap-freezing was less than 5 minutes. A systematic gross and microscopic postmortem examination was carried out. All of the animal studies were conducted under Sidney Kimmel Cancer Center and/or AntiCancer Inc. Institutional Animal Care and Use Committee-approved protocols.

Tissue processing for $m R N A$ and RNA isolation. Fresh-frozen orthotopic and subcutaneous tumors was examined using H\&E-stained frozen sections. Orthotopic tumors of all sublines exhibited similar morphology, consisting of sheets of monotonous, closely packed tumor cells with little evidence of differentiation, interrupted by only occasional zones of largely stromal components, vascular lakes, or lymphocytic infiltrates. Fragments of tumor judged free of these nonepithelial clusters were used for mRNA preparation. Frozen tissue (1-3 $\mathrm{mm} \times 1-3 \mathrm{~mm}$ ) was submerged in liquid nitrogen in a ceramic mortar and ground to powder. The frozen tissue powder was dissolved and immediately processed for mRNA isolation using a FastTract kit for mRNA extraction (Invitrogen Corp., Carlsbad, California, USA) according to the manufacturers instructions. Xenograft tissues analyzed in this study were derived from cell lines listed in Supplemental Table 2S. Two independent microarray analyses were carried out for each xenograft subtype listed in Supplemental Table 2S.

$R N A$ and $m R N A$ extraction. For gene expression analysis, cells were harvested in lysis buffer 2 hours after the last media change at $70-80 \%$ confluence, and total RNA or mRNA was extracted using the RNeasy (QIAGEN Inc., Chatsworth, California, USA) or FastTract kits (Invitrogen Corp.). Cell lines were not split more than five times prior to RNA extraction, except where noted.

Affymetrix arrays. The protocol for mRNA quality control and gene expression analysis was that recommended by Affymetrix (Santa Clara, California, USA). In brief, approximately $1 \mu \mathrm{g}$ of mRNA was reverse transcribed with an oligo(dT) primer that has a T7 RNA polymerase promoter at the $5^{\prime}$ end. Second-strand synthesis was followed by cRNA production incorporating a biotinylated base. Hybridization to Affymetrix U95Av2 arrays representing 12,625 transcripts overnight for 16 hours was followed by washing and labeling using a fluorescently labeled $\mathrm{Ab}$. The arrays were read and data processed using Affymetrix equipment and software as reported previously $(18,19)$.

Data analysis. Detailed protocols for data analysis and documentation of the sensitivity, reproducibility, and other aspects of the quantitative statistical microarray analysis using Affymetrix technology have been reported $(18,19)$. Forty to fifty percent of the surveyed genes were present according to the Affymetrix Microarray Suite 5.0 software used in these experiments. The concordance analysis of differential gene expression across the data sets was performed using Affymetrix MicroDB v. 3.0 and DMT v.3.0 software as described earlier $(18,19)$. We processed the microarray data using the Affymetrix Microarray Suite v.5.0 software and performed statistical analysis of expression data set using the Affymetrix MicroDB and Affymetrix DMT software. These analyses identified a set of 218 genes (91 upregulated and 127 downregulated transcripts) differentially regulated in tumors from patients with recurrent versus nonrecurrent prostate cancer at the statistically significant level $(P<0.05)$ defined by both the $t$ test and Mann-Whitney test (Supplemental Table 3S). 
To identify transcript abundance changes associated with highly aggressive malignant behavior of human prostate carcinoma cells in the model system, we selected three different experimental conditions for gene expression analysis in human prostate carcinoma xenografts and xenograft-derived cell lines (Supplemental Table 2S, and ref. 19). First, we compared gene expression profiles of the highly metastatic PC-3MLN4 human prostate carcinoma cell line growing in the mouse prostate (orthotopic metastasis-promoting setting) versus PC-3MLN4 xenografts growing subcutaneously (ectopic metastasissuppressing setting). Second, we compared gene expression profiles of the highly metastatic PC-3MLN4 human prostate carcinoma cell line growing in the orthotopic metastasis-promoting setting versus orthotopic xenografts derived from less-metastatic parental sublines PC-3 and PC-3M. Third, we identified genes that are differentially regulated in a concordant manner in multiple xenograft-derived human prostate carcinoma cell lines compared with the normal human prostate epithelial cells (PC-3/LNCap consensus class; ref. 19). The concordance analysis of differential gene expression across the clinical and experimental data sets defined for each experimental setting was performed using Affymetrix MicroDB v. 3.0 and DMT v.3.0 software as described earlier (19). The Pearson correlation coefficient for individual test samples and the appropriate reference standard was determined using the Microsoft Excel software (Microsoft Corp., Redmond, Washington, USA) as described in the signature discovery protocol (see signature discovery and validation protocol and Supplemental Tables 4S-6S for details).

Survival analysis. The Kaplan-Meier survival analysis was carried out using the GraphPad Prism version 4.00 software (GraphPad Software for Science Inc., San Diego, California, USA; http://www.graphpad.com). The end point for survival analysis was the biochemical recurrence defined by the serum PSA increase after therapy. The disease-free interval (DFI) was defined as the time period between the date of RP and the date of PSA relapse (recurrence group) or the date of last follow-up (nonrecurrence group). Statistical significance of the difference between the survival curves for different groups of patients was assessed using $\chi^{2}$ and log-rank tests.

\section{Results}

Identification of molecular signatures distinguishing subgroups of prostate cancer patients with distinct clinical outcome after therapy. To identify the outcome-predictor signatures, we used as a training data set the expression analysis of 12,625 transcripts in 21 prostate tumor samples obtained from patients with prostate cancer who had distinct clinical outcome after therapy. Using biochemical evidence of relapse after therapy as a criterion of treatment failure, 21 patients were divided into two subgroups representing prostate cancer with recurrent (8 patients) and nonrecurrent (13 patients) clinical behavior (14). This analysis identified a set of 218 genes (91 upregulated and 127 downregulated transcripts) differentially regulated in tumors from patients with recurrent versus nonrecurrent prostate cancer at the statistically significant level $(P<0.05)$ defined by both $t$ tests and Mann-Whitney tests (Supplemental Table 3S).

To reduce the number of hits in potential outcome predictor clusters and to identify transcripts of potential biological relevance, we compared the expression profile of 218 genes to the expression profiles of transcripts differentially regulated in multiple experimental models of human prostate cancer (19) in the search for genes with consistently concordant expression patterns across multiple data sets and various experimental conditions (see signature discovery and validation protocol presented in the supplemental data for details). We identified several small gene clusters exhibiting highly concordant pattern of expression (Pearson correlation coefficient: $r>0.95$ ) in clinical and experimental samples. We evaluated the prognostic power of each identified cluster of coregulated transcripts based on ability to segregate the patients with recurrent and nonrecurrent prostate tumors into distinct subgroups and selected a single best-performing cluster for each binary condition specified in Supplemental Table 4S (Figure 1 ; Tables 1 and 2). To assess a potential prognostic relevance of individual gene clusters, we calculated a Pearson correlation coefficient for each of 21 tumor samples by comparing the expression profiles of individual samples to the "average" expression profile of recurrent versus nonrecurrent tumors and expression profiles of relevant experimental samples (Table 1 and Figure 1). Based on expected correlation

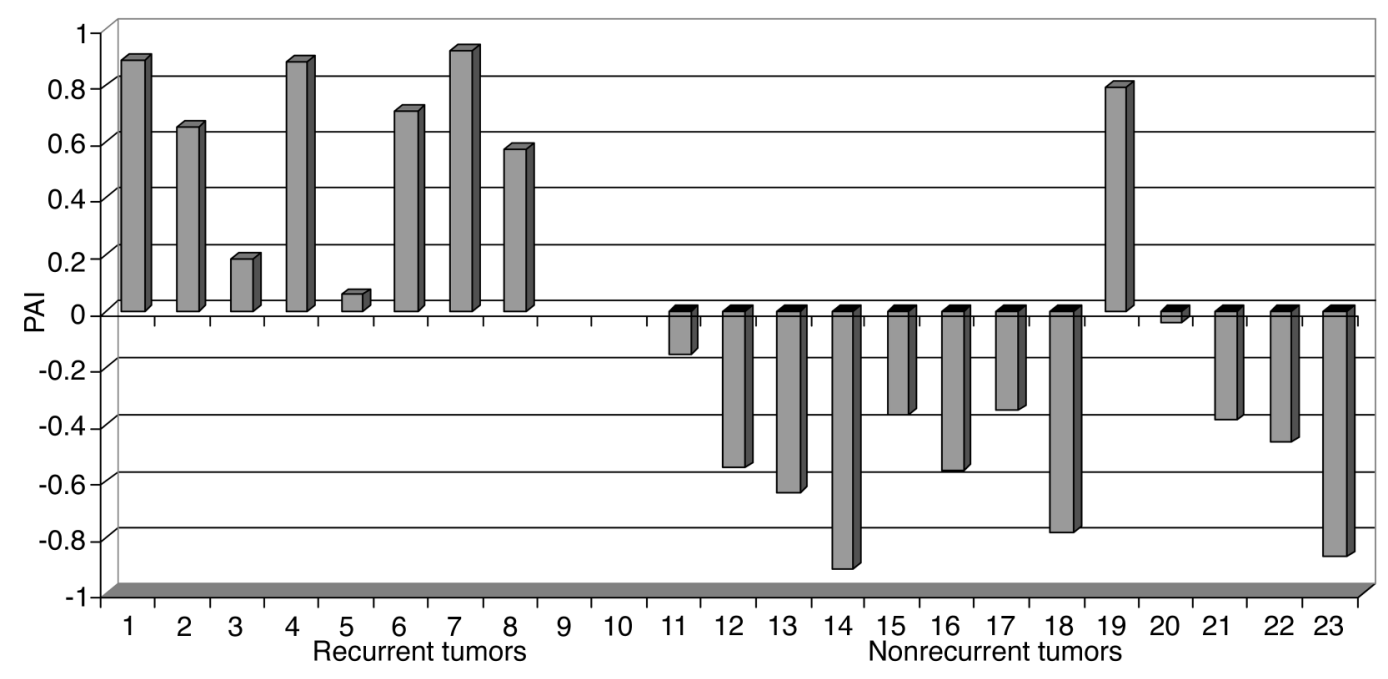

Figure 1

PAls defined by the expression profile of the prostate cancer recurrence predictor signature 1 for 21 prostate carcinoma samples constituting a signature discovery (training) data set. Prostate tumor samples were taken from the patients at the time of surgery and subjected to a microarray gene expression analysis as described in Methods. Note that all samples derived from tumors of patients who subsequently manifested a biochemical relapse of disease have positive PAI values, whereas 12 of 13 samples obtained from patients who remained disease-free have negative PAI values. See text for details. 
of expression profiles of identified gene clusters with recurrent clinical behavior of prostate cancer, we named the corresponding correlation coefficients calculated for individual samples the phenotype association indices (PAIs).

Using this strategy, we identified several gene clusters (Tables 1 and 2) discriminating with $86-95 \%$ accuracy human prostate tumors exhibiting recurrent or nonrecurrent clinical behavior (Figure 1; Tables 1 and 2). Figure 1 illustrates application of the five-gene cluster to characterize clinical prostate cancer samples according to their propensity for recurrence after therapy. The expression pattern of the genes in the recurrence predictor cluster was analyzed in each of 21 separate clinical samples. The analysis produces a quantitative PAI (plotted on the $y$ axis) for each of the 21 clinical prostate cancer samples. Tumors that are likely to recur are expected to have positive PAIs reflecting positive correlation of gene expression with metastasis-promoting orthotopic xenografts, while those that are unlikely to recur are expected to have negative association indices.

Figure 1 shows the PAIs for eight samples from patients who later had recurrence as bars $1-8$, while the association indices for 13 samples from patients whose tumors did not recur is shown as bars 11-23. Eight of the eight samples (or 100\%) from patients who later experienced recurrence had positive PAIs and so were properly classified. Twelve of the 13 samples (or 92.3\%) from patients whose tumors did not recur had negative PAIs and so were properly classified as nonrecurrent tumors. Thus, overall, 20 of the 21 samples (or 95.2\%) were properly classified using a five-gene recurrence predictor signature. Two alternative clusters identified using this strategy showed similar sample classification performance (Tables 1 and 2).

To further evaluate the prognostic power of identified gene expression signatures, we performed the Kaplan-Meier survival analysis using as a clinical end-point DFI after therapy in prostate cancer patients with positive and negative PAIs. The KaplanMeier survival curves showed a highly significant difference in the probability that prostate cancer patients would remain disease-free after therapy between the groups with positive and negative PAIs defined by the signatures (Figure 2, A-C), suggesting that patients with positive PAIs exhibit a poor-outcome signature whereas patients with negative PAIs manifest a good-outcome signature. The estimated hazard ratio for disease recurrence after therapy in the group of patients with positive PAIs as compared with the group of patients with negative PAIs defined by the recurrence predictor signature 3 (Table 1) was 9.046 (95\% confidence interval of ratio, $3.022-76.41 ; P=0.001)$. Eighty-six percent of patients with the positive PAIs had a disease recurrence within 5 years after therapy, whereas $85 \%$ of patients with the negative PAIs remained relapse-free for at least 5 years (Figure 2C). Based on this analysis, we propose to identify the group of prostate cancer patients with positive PAIs as a poor-prognosis group and the group of prostate cancer patients with negative PAIs as a goodprognosis group.

Theoretically, the recurrence predictor algorithm based on a combination of signatures should be more robust than a single predictor signature, particularly during the validation analysis using an independent test cohort of patients. Next, we analyzed whether a combination of the three signatures would perform in a patient's classification test with accuracy similar to that of the individual signatures. We found that the cut-off value of PAIs greater than 0.2 scored in two of three individual clusters allowed achievement of the $90 \%$ recurrence prediction accuracy (Table 2). This recurrence predictor algorithm correctly identified $88 \%$ of patients with recurrent disease and $92 \%$ of patients with nonrecurrent disease (Table 2). The Kaplan-Meier survival analysis (Figure 2D) showed that the median relapse-free survival after therapy of patients in the poor-prognosis group was 26 months. All patients in the poor-prognosis group had a disease recurrence within 5 years after therapy, whereas $92 \%$ of patients in the good-prognosis group remained relapse-free for at least 5 years. The estimated hazard ratio for disease recurrence after therapy in the poor-prognosis group of patients as compared with the good-prognosis group of patients defined by the recurrence predictor algorithm was 20.32 (95\% confidence interval of ratio, 6.047-158.1; $P<0.0001$ ).

Validation of the outcome predictor signatures using independent clinical data set. To validate the potential clinical utility of identified molecular signatures, we evaluated the prognostic power of signatures applied to an independent set of 79 clinical samples obtained from 37 patients with prostate cancer who developed recurrence after the therapy and 42 patients who remained disease-free (Supplemental Table 1S). The Kaplan-Meier survival analysis demonstrated that all three recurrence predictor signatures segregate prostate cancer patients into subgroups with a statistically significant difference in the probability of remaining relapse-free after the therapy (Table 3). Interestingly, application of the recurrence predictor algorithm (the cut-off value of PAIs greater than 0.2 scored in two of three individual clusters) appears to perform better than individual signatures in a patient's stratification test using an independent data set (Table 3).

The Kaplan-Meier survival analysis (Figure 3A) showed that the median relapse-free survival after therapy of patients in the poorprognosis group defined by the recurrence predictor algorithm was

Table 1

Gene expression signatures associated with recurrent prostate cancer

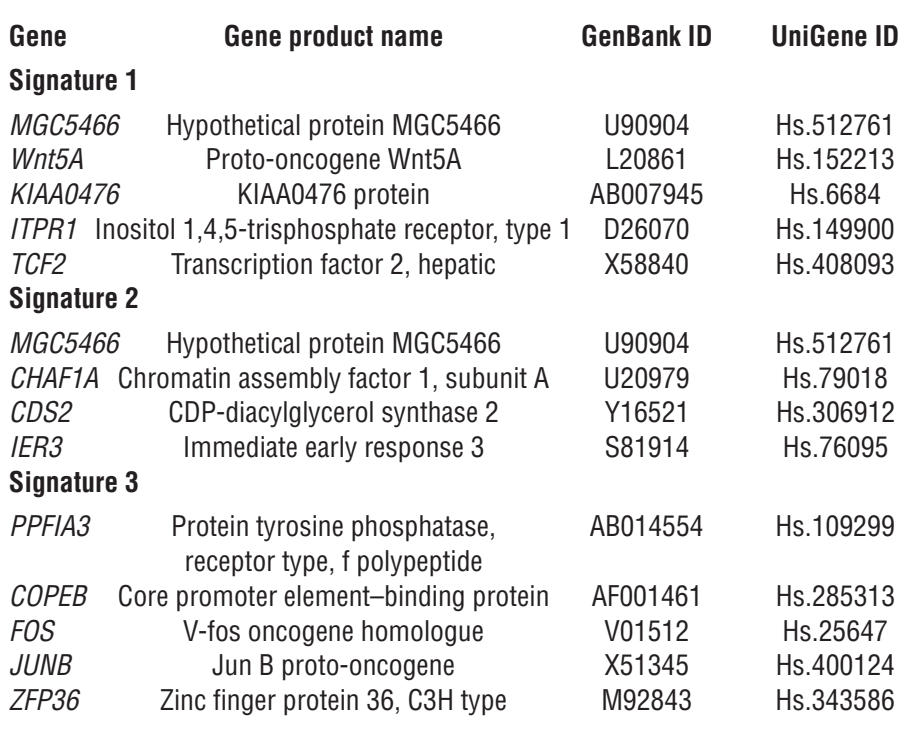

The transcripts constituting each signature were selected based on Pearson correlation coefficients $(r>0.95)$ reflecting a degree of similarity of expression profiles in clinical tumor samples (recurrent versus nonrecurrent tumors) and experimental samples. Selection of transcripts was performed from sets of genes exhibiting concordant changes of transcript abundance behavior in recurrent versus nonrecurrent clinical tumor samples (218 transcripts) and experimental conditions independently defined for each signature (signature 1: PC-3MLN4 orthotopic versus subcutaneous xenografts; signature 2: PC-3MLN4 versus PC-3M and PC-3 orthotopic xenografts; signature 3: PC-3/LNCap consensus class; ref. 19). 
Table 2

Prostate cancer recurrence prediction accuracy in a good-prognosis and a poor-prognosis subgroup of patients defined according to whether they had a good-prognosis or a poor-prognosis signature

\begin{tabular}{|c|c|c|c|c|c|}
\hline Recurrence signature & Correlation coefficient & Recurrent cancer & Nonrecurrent cancer & Overall & $P$ value \\
\hline Signature 1 & $r=0.999$ & $100 \%$ (8 of 8$)$ & $92 \%(12$ of 13$)$ & $95 \%(20$ of 21$)$ & $<0.0001$ \\
\hline Signature 2 & $r=0.963$ & $88 \%(7$ of 8$)$ & $92 \%(12$ of 13$)$ & $90 \%(19$ of 21$)$ & $<0.0001$ \\
\hline Signature 3 & $r=0.996$ & $75 \%(6$ of 8$)$ & $92 \%(12$ of 13$)$ & $86 \%(18$ of 21$)$ & 0.001 \\
\hline Algorithm & NA & $88 \%(7$ of 8$)$ & $92 \%(12$ of 13$)$ & $90 \%(19$ of 21$)$ & $<0.0001$ \\
\hline
\end{tabular}

Twenty-one prostate cancer patients constituting a signature discovery (training) data set were classified according to whether they had a good-prognosis signature or poor-prognosis signature based on PAI values defined by either individual recurrence predictor signatures or recurrence predictor algorithm, which takes into account calls from all three signatures. The number of correct predictions in poor-prognosis and good-prognosis groups is shown as a fraction of patients with the observed clinical outcome after therapy (8 patients developed relapse and 13 patients remained disease-free). Correlation coefficients reflect a degree of similarity of expression profiles in clinical tumor samples (recurrent versus nonrecurrent tumors) and experimental samples (see Table 1). $P$ values were calculated using the log-rank test and reflect the statistically significant difference in the probability that patients would remain disease-free between poor-prognosis and good-prognosis subgroups. We selected the prognosis discrimination cut-off value for each signature based on highest level of statistical significance in a patient's stratification into poor and good prognosis groups as determined by the log-rank test (lowest $P$ value and highest hazard ratio; see Supplemental Table 6S).

34.6 months. Sixty-seven percent of patients in the poor-prognosis group had a disease recurrence within 5 years after therapy, whereas $76 \%$ of patients in the good-prognosis group remained relapse-free for at least 5 years. The estimated hazard ratio for disease recurrence after therapy in the poor-prognosis group as compared with the good-prognosis group of patients defined by the recurrence predictor algorithm was 4.224 (95\% confidence interval of ratio, $2.455-9.781 ; P<0.0001)$. Overall, the application of the recurrence predictor algorithm allowed accurate stratification into the poorprognosis group $82 \%$ of patients who failed the therapy within 1 year after prostatectomy. The recurrence predictor algorithm seems to demonstrate more accurate performance in patient's classification compared with the conventional markers of outcome such as preoperative PSA level or RP Gleason sum (Figures 3 and 4; Table 4).

Recurrence predictor signatures provide additional predictive value over conventional markers of outcome. Next, we thought to determine whether the application of the recurrence predictor signatures would provide an additional predictive value when combined with conventional markers of outcome such as preoperative PSA level and Gleason score. Both preoperative PSA level and RP Gleason sum were significant predictors of prostate cancer recurrence after therapy in the validation cohort of 79 patients (Figure 3D and Figure 4C).
A

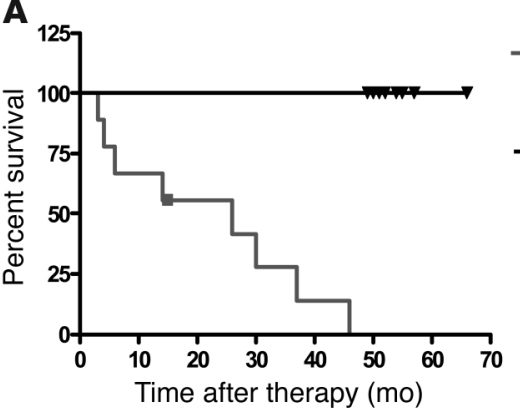

C

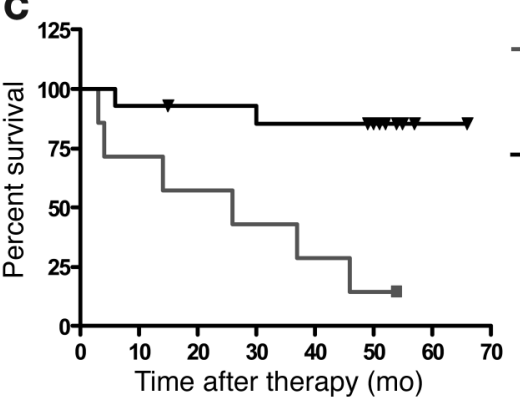

$\rightarrow-$ Positive signature 1 Median survival: 26 mo 5-year survival: $0 \%$

$\rightarrow$ Negative signature 1 5-year survival: $100 \%$ Log-rank test: $P<0.0001$

$\rightarrow-$ Positive signature 3 Median survival: 26 mo 5-year survival: $14 \%$

- Negative signature 3 5-year survival: $85 \%$ Log-rank test:

Hazard ratio: 9.046

95\% Cl: 3.022-76.41 $P=0.001$
B

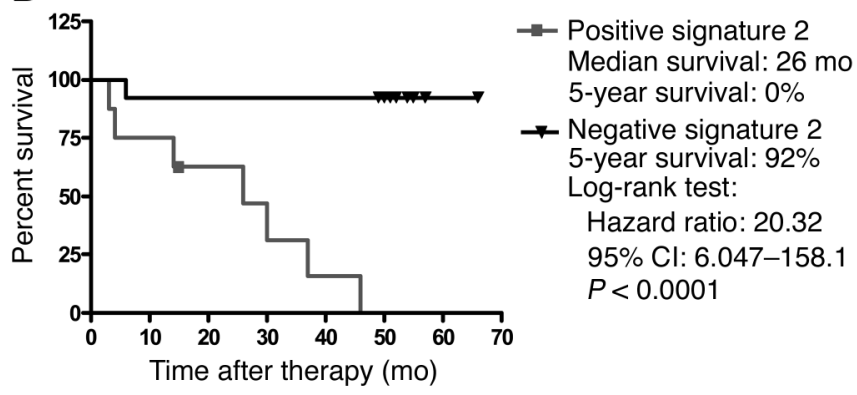

D

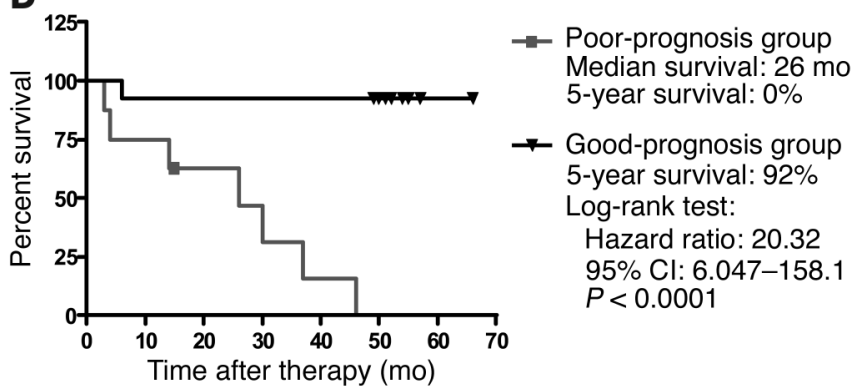

Figure 2

Kaplan-Meier analysis of the probability that patients would remain disease-free among 21 prostate cancer patients constituting a signature discovery group according to whether they had good-prognosis or poor-prognosis signatures defined by the recurrence predictor signature 1 (A), recurrence predictor signature $2(B)$, recurrence predictor signature $3(\mathbf{C})$, and the recurrence predictor algorithm, which takes into account calls from all three signatures (D). The cut-off values for each marker were identified through the detailed analysis of behavior of log-rank test $P$ values across the range of the measurements for each marker. We selected the prognosis discrimination cut-off value for each signature based on highest level of statistical significance in patients' stratification into poor- and good-prognosis groups as determined by the log-rank test (lowest $P$ value and highest hazard ratio; see Supplemental Table 6S). Cl, confidence interval. 
Table 3

Stratification of 79 prostate cancer patients based on recurrence predictor signatures into poor- and good-prognosis groups at the time of diagnosis

\begin{tabular}{lccccc}
\hline $\begin{array}{l}\text { Recurrence } \\
\text { signature }\end{array}$ & $\begin{array}{c}\text { Poor prognosis, } \\
\text { 5-year survival }\end{array}$ & $\begin{array}{c}\text { Good prognosis, } \\
\text { 5-year survival }\end{array}$ & Hazard ratio & \multicolumn{2}{c}{$\begin{array}{c}\text { 95\% Confidence } \\
\text { interval of ratio }\end{array}$} \\
Signature 1 & $41 \%$ & $78 \%$ & 2.858 & $1.405-5.143$ & \multicolumn{2}{c}{ value } \\
Signature 2 & $44 \%$ & $79 \%$ & 3.473 & $1.584-5.806$ & 0.0028 \\
Signature 3 & $41 \%$ & $76 \%$ & 3.351 & $1.810-6.907$ & 0.0008 \\
Algorithm & $33 \%$ & $76 \%$ & 4.224 & $2.455-9.781$ & $<0002$
\end{tabular}

Seventy-nine prostate cancer patients constituting a signature validation (test) data set were classified according to whether they had a good-prognosis signature or poor-prognosis signature based on PAI values defined by either individual recurrence predictor signatures or the recurrence predictor algorithm, which takes into account calls from all three signatures. Kaplan-Meier analysis was performed to evaluate the probability that patients would remain disease-free according to whether they had a poor-prognosis or a good-prognosis signature and to determine the proportion of patients who would remain disease-free at least 5 years after therapy in poor-prognosis and good-prognosis subgroups. hazard ratios, 95\% confidence intervals, and $P$ values were calculated using the log-rank test.

The Kaplan-Meier survival analysis (Figure 3D) showed that the median relapse-free survival after therapy of patients in the poor-prognosis group defined by the high preoperative PSA level was 49.0 months. Sixty percent of patients in the poor-prognosis group had a disease recurrence within 5 years after therapy, whereas $73 \%$ of patients in the good-prognosis group remained relapse-free for at least 5 years. The estimated hazard ratio for disease recurrence after therapy in the poor-prognosis group as compared with the good-prognosis group of patients defined by the preoperative PSA level was 2.551 (95\% confidence interval of ratio, 1.344-4.895; $P=0.0043$ ). Prediction of the outcome after therapy based on preoperative PSA level, however, accurately stratified into poor-prognosis group only $65 \%$ of patients who failed the therapy within 1 year after prostatectomy (Table 4).
Next, we set out to determine whether the application of the recurrence predictor algorithm would identify subgroups of patients with distinct clinical outcome after therapy in both high and low PSAexpressing groups, thus adding additional predictive value to the therapy outcome classification based on preoperative PSA level alone.

In the group of patients with high preoperative PSA level (Figure $3 \mathrm{~B})$, the median relapse-free survival after therapy of patients in the poor-prognosis subgroup defined by the recurrence predictor algorithm was 36.2 months. Seventy-three percent of patients in the poor-prognosis subgroup had a disease recurrence within 5 years after therapy. Conversely, $73 \%$ of patients in the good-prognosis subgroup remained relapse-free for at least 5 years. The estimated hazard ratio for disease recurrence after therapy in the poor-prognosis
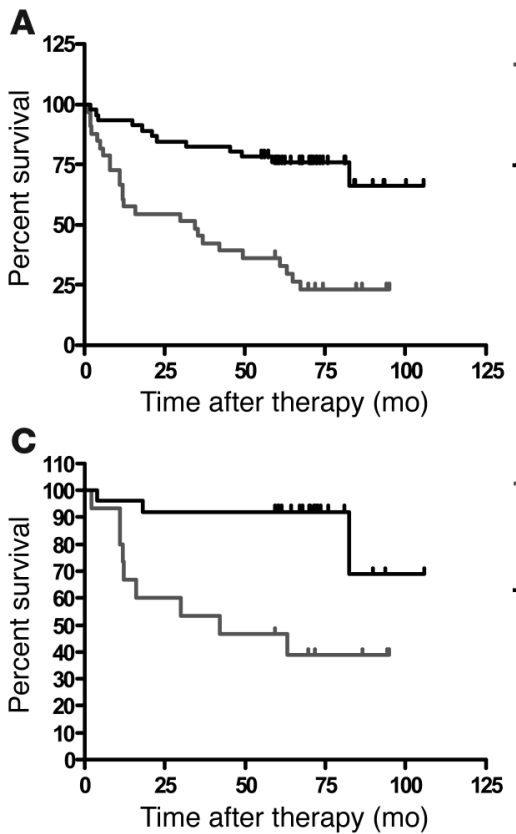

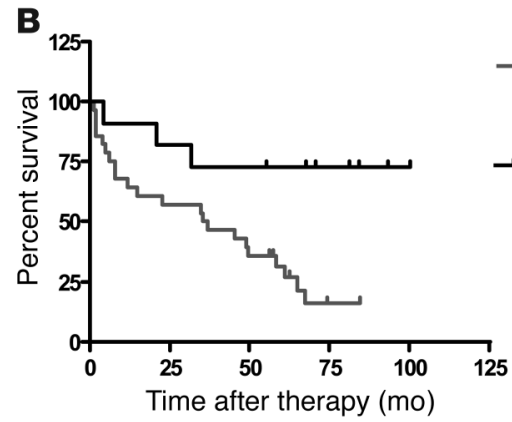
- Poor-prognosis group Median survival: 36.2 mo 5-year survival: $27 \%$ Hazard ratio: 4.315 95\% Cl: 1.338-7.025 $P=0.0081$ Median survival: 34.6 5-year survival: $33 \%$

- Good-prognosis group 5-year survival: $76 \%$ Log-rank test: Hazard ratio: 4.224 95\% Cl: 2.455-9.781 $P<0.0001$

D

Poor-prognosis group
Median survival: $42.0 \mathrm{~m}$
5-year survival: $47 \%$
- Good-prognosis group
5-year survival: $92 \%$
Log-rank test:
Hazard ratio: 6.247
$\quad 95 \% \mathrm{Cl}: 2.134-24.48$
$P=0.0015$

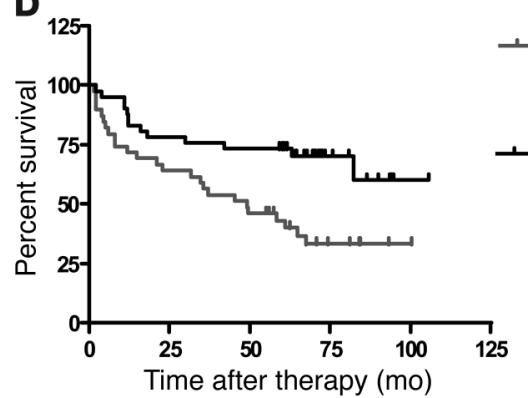

- High properative PSA Median survival: 49.0 mo 5-year survival: $40 \%$

- Low properative PSA 5-year survival: $73 \%$ Log-rank test: Hazard ratio: 2.551 95\% Cl: 1.344-4.895 $P=0.0043$

Figure 3

Kaplan-Meier analysis of the probability that patients would remain disease-free among 79 prostate cancer patients constituting a signature validation group for all patients $(\mathbf{A})$, patients with high $(\mathbf{B})$ or low $(\mathbf{C})$ preoperative PSA levels in blood according to whether they had good-prognosis or poor-prognosis signatures defined by the recurrence predictor algorithm, or whether they had high or low preoperative PSA level in the blood (D). Preoperative PSA level of $7.8 \mathrm{ng} / \mathrm{ml}$ was used as a cut-off discrimination level for patients' stratification into poor- and good-prognosis subgroups. The cut-off values for each marker were identified through the detailed analysis of behavior of log-rank test $P$ values across the range of the measurements for each marker. We selected the prognosis discrimination cut-off value for each marker based on highest level of statistical significance in patients' stratification into poor- and good-prognosis groups as determined by the log-rank test (lowest $P$ value and highest hazard ratio). 
A

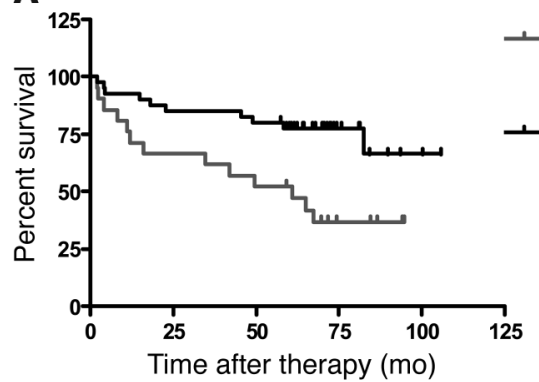

C

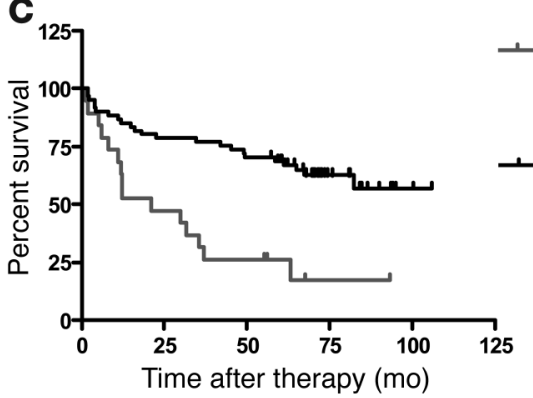

- Poor-prognosis group Median survival: $61.0 \mathrm{mo}$ 5-year survival: $47 \%$

- Good-prognosis group 5-year survival: $77 \%$ Log-rank test: Hazard ratio: 3.024 95\% Cl: 1.457-8.671 $P=0.0055$

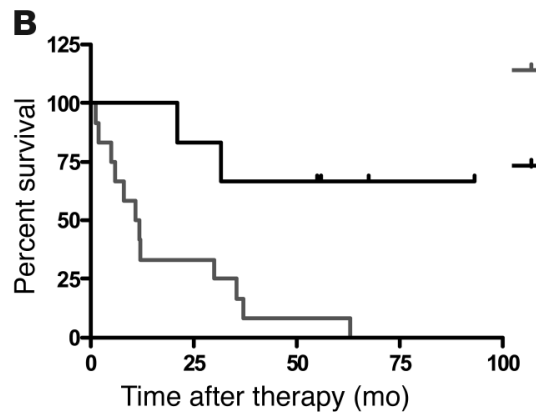

- Poor-prognosis group Median survival: $11.5 \mathrm{mo}$ 5-year survival: $0 \%$

- Good-prognosis group 5-year survival: $67 \%$ Log-rank test: Hazard ratio: 6.143 95\% Cl: 1.573-13.49 $P=0.0053$

Figure 4

Kaplan-Meier analysis of the probability that patients would remain disease-free among prostate cancer patients with Gleason sum 6 and 7 tumors (A) and patients with Gleason sum 8 and 9 tumors (B) according to whether they had good-prognosis or poor-prognosis signatures defined by the recurrence predictor algorithm or whether they had Gleason sum 8 and 9 or Gleason sum 6 and 7 prostate tumors (C).

subgroup as compared with the good-prognosis subgroup of patients defined by the recurrence predictor algorithm was 4.315 (95\% confidence interval of ratio, 1.338-7.025; $P=0.0081$ ).

In the group of patients with low preoperative PSA level (Figure $3 \mathrm{C})$, the median relapse-free survival after therapy of patients in the poor-prognosis subgroup defined by the recurrence predictor algorithm was 42.0 months. Fifty-three percent of patients in the poor-prognosis subgroup had a disease recurrence within 5 years after therapy, whereas $92 \%$ of patients in the good-prognosis subgroup remained relapse-free for at least 5 years. The estimated hazard ratio for disease recurrence after therapy in the poor-prognosis subgroup as compared with the good-prognosis subgroup of patients defined by the recurrence predictor algorithm was 6.247 (95\% confidence interval of ratio, 2.134-24.48; $P=0.0015$ ). Overall, combination of the recurrence predictor algorithm and preoperative PSA level allowed accurate stratification into poorprognosis group $88 \%$ of patients who failed the therapy within 1 year after prostatectomy (Table 4).

RP Gleason sum is a significant predictor of relapse-free survival in the validation cohort of 79 prostate cancer patients (Figure 4C). The Kaplan-Meier survival analysis (Figure 4C) demonstrates that the median relapse-free survival after therapy of patients with the RP Gleason sum 8 and 9 was 21.0 months, thus defining the poor-prognosis group based on histopathological criteria. Seventy-four percent of patients in the poor-prognosis group had a disease recurrence within 5 years after therapy, whereas $69 \%$ of patients in the goodprognosis group (RP Gleason sum 6 and 7) remained relapse-free for at least 5 years. The estimated hazard ratio for disease recurrence after therapy in the poor-prognosis group as compared with the goodprognosis group of patients defined by the RP Gleason sum criteria was 3.335 (95\% confidence interval of ratio, 2.389-13.70; $P<0.0001$ ). RP Gleason sum-based outcome classification accurately stratified into the poor-prognosis group only $47 \%$ of patients who failed the therapy within 1 year after prostatectomy (Table 4 ).

In the group of patients with RP Gleason sum 6 and 7 (Figure 4A), the median relapse-free survival after therapy of patients in the poorprognosis subgroup defined by the recurrence predictor algorithm was 61.0 months. Fifty-three percent of patients in the poor-prognosis subgroup had a disease recurrence within 5 years after therapy, whereas $77 \%$ of patients in the good-prognosis subgroup remained relapse-free for at least 5 years. The estimated hazard ratio for disease recurrence after therapy in the poor-prognosis subgroup as compared with the good-prognosis subgroup of patients defined by the recurrence predictor algorithm was 3.024 (95\% confidence interval of ratio, $1.457-8.671 ; P=0.0055)$.

In the group of patients with RP Gleason sum 8 and 9 (Figure $4 \mathrm{~B})$, the median relapse-free survival after therapy in the poorprognosis subgroup defined by the recurrence predictor algorithm was 11.5 months. One hundred percent of patients in the poorprognosis subgroup had a disease recurrence within 5 years after therapy, whereas $67 \%$ of patients in the good-prognosis subgroup remained relapse-free for at least 5 years. The estimated hazard ratio for disease recurrence after therapy in the poor-prognosis subgroup as compared with the good-prognosis subgroup of patients defined by the recurrence predictor algorithm was 6.143 (95\% confidence interval of ratio, $1.573-13.49 ; P=0.0053$ ). Overall, a patient's classification using a combination of the recurrence predictor algorithm and RP Gleason sum allowed an accurate stratification into the poor-prognosis group $82 \%$ of patients who failed the therapy within 1 year after prostatectomy (Table 4). Based on this analysis, we concluded that application of the recurrence predictor algorithm appears to provide an additional predictive value to the therapy outcome classification based on established markers of prostate cancer outcome. 


\title{
Table 4
}

Prostate cancer recurrence prediction accuracy in poor-prognosis and good-prognosis subgroups of patients defined by a gene expression-based recurrence predictor algorithm alone or in combination with established biochemical and histopathological markers of outcome

\begin{tabular}{|c|c|c|c|c|}
\hline Recurrence predictor & Recurrent cancer & Nonrecurrent cancer & Year 1 recurrence & Overall \\
\hline Recurrence algorithm & $68 \%(25$ of 37$)$ & $81 \%$ (34 of 42$)$ & $82 \%(14$ of 17$)$ & $75 \%$ (59 of 79$)$ \\
\hline PSA & $68 \%(25$ of 37$)$ & $67 \%$ (28 of 42$)$ & $65 \%(11$ of 17$)$ & $67 \%(53$ of 79$)$ \\
\hline PSA and algorithm & $84 \%$ (31 of 37$)$ & $71 \%(30$ of 42$)$ & $88 \%(15$ of 17$)$ & $77 \%(61$ of 79$)$ \\
\hline RP Gleason sum & $38 \%(14$ of 37$)$ & $90 \%$ (38 of 42$)$ & $47 \%$ (8 of 17$)$ & $66 \%(52$ of 79$)$ \\
\hline RP Gleason sum and algorithm & $68 \%(25$ of 37$)$ & $81 \%$ (34 of 42$)$ & $82 \%(14$ of 17$)$ & $75 \%$ (59 of 79$)$ \\
\hline PSA and RP Gleason sum & $81 \%$ (30 of 37$)$ & $67 \%$ (28 of 42$)$ & $82 \%(14$ of 17$)$ & $73 \%(58$ of 79$)$ \\
\hline Nomogram & $62 \%(23$ of 37$)$ & $79 \%$ (33 of 42$)$ & $71 \%(12$ of 17$)$ & $71 \%(56$ of 79$)$ \\
\hline Nomogram and algorithm & $68 \%$ (25 of 37$)$ & $81 \%$ (34 of 42$)$ & $82 \%(14$ of 17$)$ & $75 \%$ (59 of 79$)$ \\
\hline
\end{tabular}

\begin{abstract}
Seventy-nine prostate cancer patients constituting a signature validation (test) data set were classified according to whether they had a good-prognosis signature or poor-prognosis signature based on PAI values defined by either individual recurrence predictor signatures or the recurrence predictor algorithm, which takes into account calls from all three signatures. The number of correct predictions in poor-prognosis and good-prognosis groups is shown as a fraction of patients with the observed clinical outcome after therapy (37 patients developed relapse and 42 patients remained disease-free). PSA and Gleason sum cut-off values for segregation of poor-prognosis and good-prognosis subgroups were defined to achieve the most accurate and statistically significant recurrence prediction in this cohort of patients. Multiparameter nomogram-based prognosis predictor was defined as described in Methods using $50 \%$ relapse-free survival probability as a cut-off for patients' stratification into poor- and good-prognosis subgroups.
\end{abstract}

In the Cox multivariate proportional hazard analysis, we included in the model four variables that were identified as statistically significant prostate cancer recurrence predictors in a cohort of 79 patients using a univariate survival analysis: the preoperative PSA levels, the age of patients at the time of diagnosis, the RP Gleason sum, and the gene expression-based recurrence predictor algorithm. The multivariate analysis demonstrated that both the recurrence predictor algorithm $(P=0.0001)$ and RP Gleason sum $(P=0.0306)$ remained statistically significant prognostic markers, whereas preoperative PSA levels $(P=0.0593)$ and age $(P=0.0956)$ failed to achieve the $P=0.05$ level of significance in patients' stratification (Supplemental Table 7S). Interestingly, the recurrence predictor algorithm $[\mathrm{HR}=4.0124(1.9985-8.0556) ; P=0.0001]$ (HR, hazard ratio) appears to perform better in selecting poorprognosis patients than the RP Gleason sum $[\mathrm{HR}=1.5367$ (1.040-2.2684); $P=0.0306]$.

Recurrence predictor signatures provide additional predictive value over outcome prediction based on a multiparameter nomogram. Classification nomograms are generally recognized as the most efficient clinically useful models currently available for prediction of the probability of relapse-free survival after therapy of individual prostate cancer patients (4-7). We applied the Kattan nomogram using multiple postoperative parameters (7) for prognosis prediction classification in the test group of 79 prostate cancer patients.
A
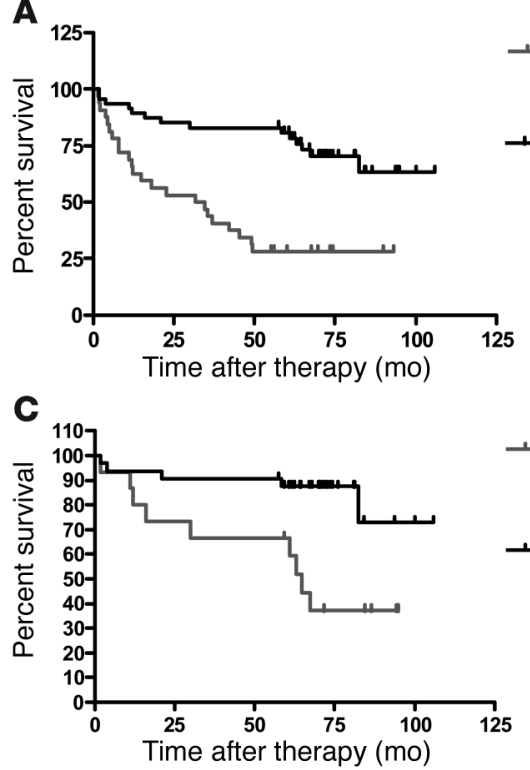

- Poor-prognosis group Median survival: $33.1 \mathrm{mo}$ 5-year survival: $28 \%$

$\rightarrow$ Good-prognosis group 5-year survival: $81 \%$ Log-rank test:

Hazard ratio: 3.757 95\% Cl: 2.318-9.647 $P<0.0001$

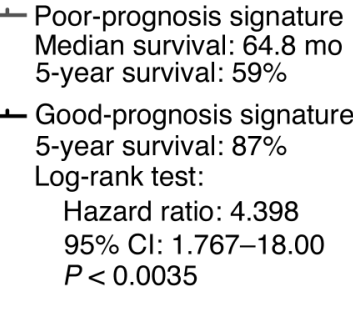

B

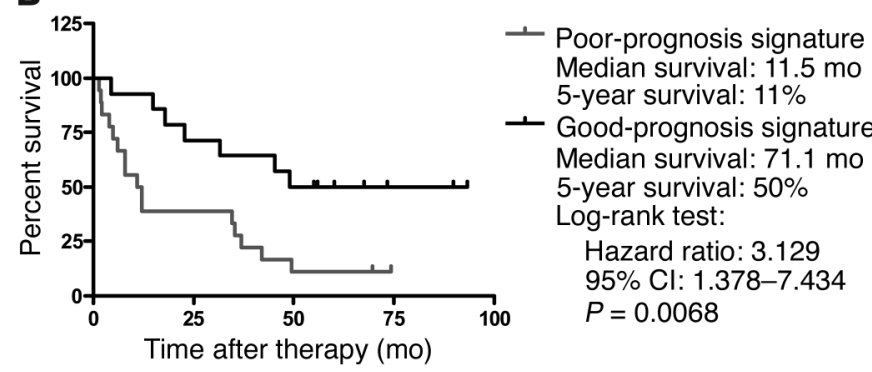

Figure 5

Kaplan-Meier analysis of the probability that patients would remain disease-free among 79 prostate cancer patients constituting a signature validation group for all patients (A), patients with poor prognosis (B), or good prognosis (C), defined by the Kattan nomogram according to whether they had a good-prognosis or poor-prognosis signatures defined by the recurrence predictor algorithm (B and $\mathbf{C}$ ) or whether they had poor or good prognosis defined by the Kattan nomogram (A). 


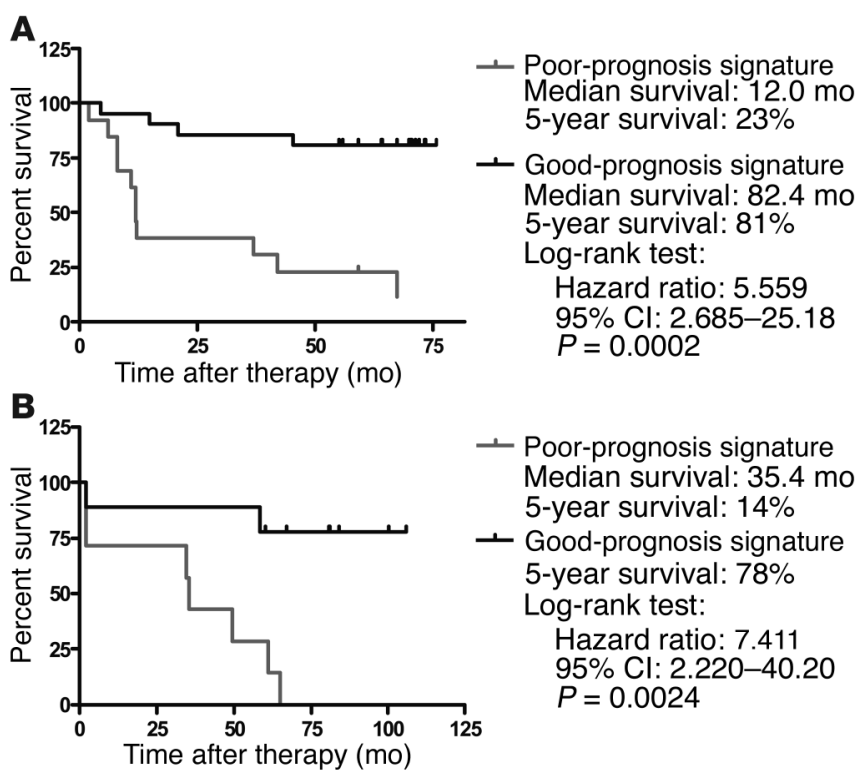

Figure 6

Kaplan-Meier analysis of the probability that patients would remain diseasefree among prostate cancer patients with stage $1 \mathrm{C}$ tumors $(\mathbf{A})$ and patients with stage $2 A$ tumors $(B)$ according to whether they had a good-prognosis or poor-prognosis signatures defined by the recurrence predictor algorithm.

The Kaplan-Meier survival analysis (Figure 5A) showed that the median relapse-free survival after therapy of patients in the poorprognosis group defined by the Kattan nomogram was 33.1 months. Seventy-two percent of patients in the poor-prognosis group had a disease recurrence within 5 years after therapy, whereas $81 \%$ of patients in the good-prognosis group remained relapse-free for at least 5 years. The estimated hazard ratio for disease recurrence after therapy in the poor-prognosis group as compared with the goodprognosis group of patients defined by the Kattan nomogram was 3.757 (95\% confidence interval of ratio, 2.318-9.647; $P<0.0001$ ). Prediction of the outcome after therapy based on Kattan nomogram accurately stratified into the poor-prognosis group $71 \%$ of patients who failed the therapy within 1 year after prostatectomy (Table 4).

Next, we thought to determine whether the application of the recurrence predictor algorithm would identify subgroups of patients with distinct clinical outcome after therapy in both poor- and goodprognosis groups defined by the Kattan nomogram, thus adding additional predictive value to the therapy outcome classification based on nomogram alone.

In the poor-prognosis group of patients defined by the Kattan nomogram the application of the recurrence predictor algorithm appears to identify two subgroups of patients with statistically significant difference in the probability of remaining relapse-free after therapy (Figure 5B). Median relapse-free survival after therapy of patients in the poor-prognosis subgroup defined by the recurrence predictor algorithm was 11.5 months compared with median relapse-free survival of 71.1 months in the good-prognosis subgroup (Figure 5B). Eighty-nine percent of patients in the poor-prognosis subgroup had a disease recurrence within 5 years after therapy. Conversely, $50 \%$ of patients in the good-prognosis subgroup remained relapse-free for at least 5 years. The estimated hazard ratio for disease recurrence after therapy in the poor-prognosis subgroup as compared with the good-prognosis subgroup of patients defined by the recurrence predictor algorithm was 3.129 (95\% confidence interval of ratio, $1.378-7.434 ; P=0.0068$ ).

Similarly, in the good-prognosis group of patients identified based on application of the Kattan nomogram, the recurrence predictor algorithm seems to define two subgroups of patients with a statistically significant difference in the probability of remaining relapsefree after therapy (Figure 5C). Median relapse-free survival after therapy of patients in the poor-prognosis subgroup defined by the recurrence predictor algorithm was 64.8 months. Forty-one percent of patients in the poor-prognosis subgroup had a disease recurrence within 5 years after therapy. Conversely, $87 \%$ of patients in the goodprognosis subgroup remained relapse-free for at least 5 years. The estimated hazard ratio for disease recurrence after therapy in the poor-prognosis subgroup as compared with the good-prognosis subgroup of patients defined by the recurrence predictor algorithm was 4.398 (95\% confidence interval of ratio, 1.767-18.00; $P=0.0035$ ). Overall, combination of the recurrence predictor algorithm and Kattan nomogram allowed accurate stratification into the poor-prognosis group $82 \%$ of patients who failed the therapy within 1 year after prostatectomy (Table 4).

Recurrence predictor algorithm defines poor- and good-prognosis subgroups of patients diagnosed with the early stage prostate cancer. Identification of subgroups of patients with distinct clinical outcome after therapy would be particularly desirable in a cohort of patients diagnosed with early-stage prostate cancer. Next, we investigated whether recurrence predictor signatures would be useful in defining subgroups of patients diagnosed with early-stage prostate cancer and having a statistically significant difference in the likelihood of disease relapse after therapy.

In the group of patients diagnosed with the stage $1 \mathrm{C}$ prostate cancer (Figure 6A), the median relapse-free survival time after therapy in the poor-prognosis subgroup defined by the recurrence predictor algorithm was 12 months. In contrast, the median relapse-free survival time after therapy in the good-prognosis group was 82.4 months. Seventyseven percent of patients in the poor-prognosis subgroup had a disease recurrence within 5 years after therapy. Conversely, $81 \%$ of patients in the good-prognosis subgroup remained relapse-free for at least 5 years. The estimated hazard ratio for disease recurrence after therapy in the poor-prognosis subgroup as compared with the good-prognosis subgroup of patients defined by the recurrence predictor algorithm was 5.559 (95\% confidence interval of ratio, 2.685-25.18; $P=0.0002$ ).

In the group of patients diagnosed with the stage $2 \mathrm{~A}$ prostate cancer (Figure 6B), the median relapse-free survival after therapy in the poorprognosis subgroup defined by the recurrence predictor algorithm was 35.4 months. Eighty-six percent of patients in the poor-prognosis subgroup had a disease recurrence within 5 years after therapy, whereas $78 \%$ of patients in the good-prognosis subgroup remained relapse-free for at least 5 years. The estimated hazard ratio for disease recurrence after therapy in the poor-prognosis subgroup as compared with the good-prognosis subgroup of patients defined by the recurrence predictor algorithm was 7.411 (95\% confidence interval of ratio, 2.220-40.20; $P=0.0024)$. Based on this analysis we concluded that application of the recurrence predictor algorithm seems to provide potentially useful clinical information in stratification of patients diagnosed with the earlystage prostate cancer into subgroups, with statistically significant difference in the likelihood of disease recurrence after therapy.

\section{Discussion}

As a result of the broad application of measurements of PSA level in the blood for early detection of prostate cancer in the United States, 
an increasing proportion of prostate cancer patients are diagnosed with early-stage tumors that are apparently confined to the prostate gland, and many patients have seemingly indolent disease not affecting an individual's survival (20). A considerable clinical heterogeneity of the early-stage prostate cancer represents a highly significant health care and socioeconomic challenge because prostate cancer is expected to be diagnosed in approximately 200,000 individuals every year (21). Consequently, it can be argued that, unlike other types of cancer, development of efficient prognostic tests rather than early detection is critical for improvement of clinical decision making and management of prostate cancer.

We hypothesized that clinically relevant genetic signatures could be found by searching for clusters of coregulated genes that display highly concordant transcript abundance behavior across multiple experimental models and clinical settings that are modeling or representing malignant phenotypes of interest $(19,22,23)$. Thus, according to this model, the primary criterion in transcript selection process should be the concordance of changes in expression rather the magnitude of changes (e.g., fold change). One of the predictions of this model is that transcripts of interest would be expected to have a tightly controlled rank order of expression within a cluster of coregulated genes, reflecting a balance of upregulated and downregulated mRNAs as a desired regulatory end point in a cell. A degree of resemblance between a test sample and reference standard in the transcript abundance rank order within a gene cluster is measured by a Pearson correlation coefficient and designated as a PAI.

Using this strategy, we discovered and validated a prostate cancer recurrence predictor algorithm that appears suitable for stratification of patients at the time of diagnosis into poor- and good-prognosis subgroups with a statistically significant difference in the disease-free survival after therapy. The algorithm is based on application of gene expression signatures associated with biochemical recurrence of prostate cancer. The signatures were defined using clusters of coregulated genes exhibiting highly concordant expression profiles $(r>0.95)$ in metastatic nude mouse models of human prostate carcinoma and tumor samples from patients with recurrent prostate cancer.

Few previous studies have applied oligonucleotide or cDNA microarrays for identification of gene expression signatures associated with biochemical recurrence of human prostate cancer $(9,14$, $16,17)$. One of the major deficiencies of these studies that somewhat limited their significance was that a single clinical data set was used for both signature discovery and validation. To our knowledge, our work is the first genomewide expression-profiling study of human prostate cancer that uses one clinical data set for signature discovery and algorithm development and a second independent data set for validation of the prostate cancer recurrence predictor algorithm.

One of the interesting features of the prostate cancer recurrence predictor algorithm described here is that it seems to provide an additional predictive value over conventional markers of outcome such as preoperative PSA level and Gleason sum. Another important feature of the identified recurrence predictor algorithm is its apparent ability to stratify patients diagnosed with the early-stage prostate cancer into subgroups with a statistically distinct likelihood of biochemical relapse after therapy. Importantly, the recurrence predictor algorithm segregates into the poor-prognosis group $88 \%$ of patients who subsequently developed disease recurrence within 1 year after prostatectomy. Based on this analysis we concluded that genetic signatures identified in this study appear to have a significant potential for development of highly accurate clinical prognostic tests suitable for stratification of prostate cancer patients at the time of diagnosis with respect to likelihood of negative or positive clinical outcome after therapy.

The causal genetic, molecular, and biological distinctions between prostate tumors with recurrent and indolent clinical behavior remain largely unknown. Our results provide, to our knowledge, the first experimental evidence of a clinically relevant transcriptional resemblance between metastatic human prostate carcinoma xenografts growing orthotopically in nude mice and primary prostate tumors from patients that subsequently developed a biochemical relapse after therapy. Our study provides a model for investigation of the potential functional relevance of identified transcriptional aberrations and suggests that genetically defined metastasis-promoting features of primary tumors seem to be one of the major contributing factors of aggressive clinical behavior and unfavorable prognosis in prostate cancer patients. This conclusion is consistent with results of the several recent studies aimed at definition of metastasis-predictor signatures in the primary human tumors representing multiple types of epithelial cancers (24-26). Our results suggest that subgroups of prostate cancer patients with poor- and good-prognosis gene expression signatures may reflect the presence of two genetically defined subtypes of human prostate carcinoma manifesting a dramatic statistically significant difference in response to therapy and, possibly, a clinically distinct course of disease progression.

One of the dominant views of prostate cancer pathogenesis is the concept of progression from hormone-dependent early-stage prostate cancer to hormone-refractory metastatic late-stage disease, with the apparent implication of increased proportion of patients with poorprognosis at the advanced stage of progression. In our validation data set of 79 samples, however, the actual frequency of recurrence remains relatively constant among the patients with different stages of prostate cancer: $47 \%$ (16 of 34 ) in stage 1C; $56 \%$ (9 of 16) in stage 2A; and $41 \%$ (12 of 29 ) in stages $2 \mathrm{~B} / 2 \mathrm{C} / 3 \mathrm{~A}$. These data suggest that progression of the disease occurs only in a subgroup of patients. Interestingly, in a subgroup of patients with good-prognosis signatures, the frequency of recurrence appears to increase in the patients with the late-stage prostate cancer: $24 \%$ (5 of 21) in stage 1C; $22 \%$ (2 of 9) in stage $2 \mathrm{~A} ; 33 \%$ ( 3 of 9 ) in stage $2 \mathrm{~B} ; 40 \%$ ( 2 of 5 ) in stage $2 \mathrm{C} / 3 \mathrm{~A}$. These results seem to imply that patients with the good-prognosis signatures may represent a subgroup undergoing a classical prostate cancer progression with a gradual increase in malignant potential. The patients with poor-prognosis signatures may represent a genetically and biologically distinct subtype of prostate cancer exhibiting highly malignant behavior at the early stage of disease with the frequency of recurrence $85 \%$ (11 of 13 ) in stage $1 \mathrm{C}$ and $100 \%$ (7 of 7 ) in stage $2 \mathrm{~A}$ patients. Activation of the Wnt signaling cascade associated with increased expression of Wnt $5 \mathrm{~A}$ and a concomitant decreased expression of a candidate tumor suppressor gene KFL6 (COPEB) mutated in prostate cancer $(27,28)$ may represent two signature pathways of the early-stage poor-prognosis subtype of human prostate cancer. Interestingly, activation of the Wnt cascade is necessary for stem cell maintenance and self-renewal $(29,30)$, suggesting that tumors from patients with the early-stage poor-prognosis subtype of human prostate cancer may be enriched for cells resembling, in part, the transcriptional program of stem cells.

In summary, using expression profiles of highly metastatic models of human prostate cancer in nude mice as a predictive reference of expected transcript abundance behavior in recurrent prostate tumors, we identified and validated the recurrence predictor signatures of human prostate cancer. The prostate cancer recurrence predictor signatures seem to provide an additional predictive value to the conventional markers of outcome and may prove to be clinically useful in 
stratification of prostate cancer patients into subgroups with distinct clinical manifestation of disease and different response to therapy.

\section{Acknowledgments}

We thank C. Pettaway (MD Anderson Cancer Center) for providing human prostate cancer cell lines and W. Sellers for providing the Affymetrix CEL files of human prostate tumors and associated clinical information. We thank M. McClelland and J. Welsh for critical comments, technical and material assistance, and help with data analysis. We thank Ann Sawyers (Sidney Kimmel Cancer Center Tumor Bank) for excellent technical assistance. This work was supported in part by an NIH/NCI grant 5RO1 CA-89827 (to G.V. Glin- sky), NIH grant UO1 CA-84999 (to W.L. Gerald), and National Cancer Institute Grant 1 R43 CA-89779 (to AntiCancer Inc.).

Received for publication September 12, 2003, and accepted in revised form December 16, 2003.

Address correspondence to: Gennadi V. Glinsky, Sidney Kimmel Cancer Center, 10835 Altman Row, San Diego, California 92121, USA. Phone: (858) 450-5990; Fax: (858) 623-2740; E-mail: gglinsky@skcc.org.

Gennadi V. Glinsky and William L. Gerald contributed equally to this work.
1. Holmberg, L., et al. 2002. A randomized trial comparing radical prostatectomy with watchful waiting in early prostate cancer. N. Engl. J. Med. 347:781-789.

2. Thomas, G.V., and Loda, M. 2002. Molecular staging of prostate cancer. In Prostate cancer principles e practice. P.W. Kantoff, P.R. Carroll, and A.V. D'Amico, editors. Lippincott Williams \& Wilkins. Philadelphia, Pennsylvania, USA. 287-303.

3. DeMarzo, A.M., Nelson, W.G., Isaacs, W.B., and Epstein, J.I. 2003. Pathological and molecular aspects of prostate cancer. Lancet. 361:955-964.

4. Kattan, M.W., Eastham, J.A., Stapleton, A.M., Wheeler, T.M., and Scardinom, P.T. 1998. A preoperative nomogram for disease recurrence following radical prostatectomy for prostate cancer. J. Natl. Cancer Inst. 90:766-771.

5. D'Amico, A.V., et al. 1999. Pretreatment nomogram for prostate-specific antigen recurrence after radical prostatectomy or external-beam radiation therapy for clinically localised prostate cancer. J. Clin. Oncol. 17:168-172.

6. Graefen, M., et al. 1999. Early prostate-specific antigen relapse after radical retropubic prostatectomy: prediction on the basis of preoperative and postoperative tumor characteristics. Eur. Urol. 36:21-30.

7. Kattan, M.W., Wheeler, T.M., and Scardino, P.T. 1999. Postoperative nomogram for disease recurrence after radical prostatectomy for prostate cancer. J. Clin. Oncol. 17:1499-1507.

8. Magee, J.A., et al. 2001. Expression profiling reveals hepsin overexpression in prostate cancer. Cancer Res. 61:5692-5696.

9. Dhanasekaran, S.M., et al. 2001. Delineation of prognostic biomarkers in prostate cancer. Nature. 412:822-826.

10. Welsh, J.B., et al. 2001. Analysis of gene expression identifies candidate markers and pharmacological targets in prostate cancer. Cancer Res. 61:5974-5978.

11. Luo, J., et al. 2001. Human prostate cancer and benign prostatic hyperplasia: molecular dissection by gene expression profiling. Cancer Res. 61:4683-4688.

12. Stamey, T.A., et al. 2001. Molecular genetic profiling of Gleason grade $4 / 5$ prostate cancers compared to benign prostatic hyperplasia. J. Urol. 166:2171-2177.

13. Luo, J., et al. 2002. Gene expression signature of benign prostatic hyperplasia revealed by cDNA microarray analysis. Prostate. 51:189-200.

14. Singh, D., et al. 2002. Gene expression correlates of clinical prostate cancer behavior. Cancer Cell. 1:203-209.

15. Rhodes, D.R., Barrette, T.R., Rubin, M.A., Ghosh, D. and Chinnaiyan, A.M. 2002. Meta-analysis of microarrays: interstudy validation of gene expression profiles reveals pathways dysregulation in prostate cancer. Cancer Res. 62:4427-4433.

16. Varambally, S., et al. 2002. The polycomb group protein EZH2 is involved in progression of prostate cancer. Nature. 419:624-629.

17. Henshall, S.M., et al. 2002. Survival analysis of genome-wide gene expression profiles of prostate cancers identifies new prognostic targets of disease relapse. Cancer Res. 63:4196-4203.

18. LaTulippe, E., et al. 2002. Comprehensive gene expression analysis of prostate cancer reveals distinct transcriptional programs associated with metastasis. Cancer Res. 62:4499-4506.

19. Glinsky, G.V., Krones-Herzig, A., Glinskii, A.B., and Gebauer, G. 2003. Microarray analysis of xenograftderived cancer cell lines representing multiple experimental models of human prostate cancer. $\mathrm{Mol}$. Carcinog. 37:209-221.

20. Potosky, A., Feuer, E., and Levin, D. 2001. Impact of screening on incidence and mortality of prostate cancer in the United States. Epidemiol. Rev. 23:181-186.

21. Greenlee, R.T., Hill-Hamon, M.B., Murray, T., and Thun, M. 2001. Cancer statistics, 2001. CA Cancer J. Clin. 51:15-36.

22. Glinsky, G.V., Krones-Herzig, A., and Glinskii, A.B. 2003. Malignancy-associated regions of transcriptional activation: gene expression profiling identifies common chromosomal regions of a recurrent transcriptional activation in human prostate, breast, ovarian, and colon cancers. Neoplasia. 5:221-228.

23. Glinsky, G.V., Ivanova, Y.A., and Glinskii, A.B. 2003. Common malignancy-associated regions of transcriptional activation (MARTA) in human prostate, breast, ovarian, and colon cancers are targets for DNA amplification. Cancer Lett. 201:67-77.

24. van 't Veer, L.J., et al. 2002. Gene expression profiling predicts clinical outcome of breast cancer. Nature. 415:530-536.

25. van de Vijver, M.J., et al. 2002. A gene expression signature as a predictor of survival in breast cancer. N. Engl. J. Med. 347:1999-2009.

26. Ramaswamy, S., Ross, K.N., Lander, E.S., and Golub, T.R. 2003. A molecular signature of metastasis in primary solid tumors. Nat. Genet. 33:49-54.

27. Narla, G., et al. 2001. KFL6, a candidate tumor suppressor gene mutated in prostate cancer. Science. 294:2563-2566.

28. Chen, C., et al. 2003. Deletion, mutation, and loss of expression of KLF6 in human prostate cancer. Am.J. Pathol. 162:1349-1354.

29. Reya, T., et al. 2003. A role for Wnt signaling in selfrenewal of haematopoietic stem cells. Nature. 423:409-414.

30. Willert, K., et al. 2003. Wnt proteins are lipid-modified and can act as stem cell growth factor. Nature. 423:448-452. 